\title{
Diatomeas de Texcala, Puebla
}

\author{
Claudia lbarra Vázquez y Eberto Novelo \\ Laboratorio de Ficología, Facultad de Ciencias, Universidad Nacional Autónoma de México \\ Apartado postal 70-620, Coyoacán, 04510 México D.F., México \\ correo electrónico: enm@hp.fciencias.unam.mx
}

\begin{abstract}
Resumen. Se registran 57 taxa específicos e infraespecíficos pertenecientes a 17 géneros de diatomeas provenientes de un arroyo en Texcala, cerca de la Ciudad de Tehuacán, Puebla. Seis taxa que no habían sido registrados previamente para México se ilustran y se describen con anotaciones de algunas características ecológicas y su distribución geográfica. Los nuevos registros son: Cocconeis scutellum var. parva (Grunow) Cleve, Cymbella turgidula Grunow, Navicula recens Lange Bertalot, Pleurosigma salinarum (Grunow), Nitzschia calida Grunow, Nitzschia constricta (Kützing) Ralfs.

Palabras clave: Bacillariophyceae, diatomeas continentales, Puebla.

Abstract. 57 species and infraspecific taxa belong to 17 genus of diatoms from a creek in Texcala, near Tehuacan City, Puebla, Mexico are registered. Six taxa not reported before from Mexico are illustrated and described with notes of some ecological characteristics and their geographical distribution. The new records are: Cocconeis scutellum var. parva (Grunow) Cleve, Cymbella turgidula Grunow, Navicula recens Lange Bertalot, Pleurosigma salinarum (Grunow), Nitzschia calida Grunow, Nitzschia constricta (Kützing) Ralfs.
\end{abstract}

Key words: Bacillariophyceae, continental diatoms, Puebla.

$\mathrm{E}$ 1 conocimiento de la flora diatomológica de agua dulce de México ha recibido poca atención por parte de los ficólogos y limnólogos. Los trabajos más extensos sobre este grupo se refieren a diatomeas fósiles (Hutchinson et al., 1956; Bradbury, 1971; Metcalfe, 1985) y en ellos se mencionan cerca de 500 especies. Los trabajos sobre diatomeas recientes también son escasos y en conjunto registran cerca de 450 especies (Ortega, 1984; Metcalfe, 1988; Tavera y González, 1990 y Tavera et al., 1994; Cantoral, 1994). Sin embargo, muy pocos de esos trabajos incluyen las descripciones e ilustraciones adecuadas para certificar las especies que se nombran para el país. Así, podemos reunir cerca de 750 taxa específicos e infraespecíficos de diatomeas de agua dulce para México, de las cuales 330 fueron registradas por Metcalfe (1988). El incremento más reciente en el conocimiento de las diatomeas dulceacuícolas se debe a tesis y no a publicaciones formales. (Quintana, 1961; Ávila, 1985; Navarro, 1988; Ávila, 1989; Cantoral, 1990; Carmona, 1990; Ibarra, 1992; Valadez, 1992; Cantoral,
1993; Cuesta 1993), y sólo un pequeño número de ellas registra especies de diatomeas para el Valle de Tehuacán (Ávila, 1985; Navarro, 1988; Ávila, 1989; Ibarra, 1992; Cuesta, 1993), en las que en conjunto se revisan 44 muestras y se registran 94 especies, la mayoría de ellas son de amplia distribución mundial y presentes en condiciones ambientales de salobres a salinas. De estas especies, sólo el $2 \%$ corresponde al municipio de Texcala y el resto a los municipios de San Lorenzo, San Hipólito Xochiltenango, Teotitlán, Zicastla y Francisco I. Madero.

El propósito de este trabajo es registrar y documentar las especies de diatomeas que encontramos en Texcala, particularmente aquellas que son nuevos registros para nuestro país, y contribuir de este modo a la documentación de la flora ficológica de la cuenca del Río Papaloapan y al estudio de comunidades de ambientes lóticos. Hemos utilizado la sistemática propuesta por Krammer y Lange Bertalot (1986, 1988, 1991a, 1991b), como criterio principal en la asignación de nombres específicos y genéricos. 


\section{Descripción del área de estudio}

San Antonio Texcala es parte del Municipio de Zapotitlán de las Salinas, al oeste de la Ciudad de Tehuacán, Puebla. Es una zona con clima extremoso en donde la vegetación vascular está representada principalmente por cardonales, tetecheras y matorrales espinosos, que forman parte de la región xerofítica mexicana (Rzedowski, 1978).

$\mathrm{El}$ arroyo de donde proviene el material estudiado aflora aproximadamente a dos kms de la carretera Tehuacán - Zapotitlán de las Salinas, cerca del poblado de San Antonio Texcala, con una localización de $18^{\circ} 24^{\prime} 30^{\prime \prime} \mathrm{N}$ y $97^{\circ} 25^{\prime} 21^{\prime \prime}$ O y con una altitud de 1560 a $1600 \mathrm{msnm}$. (figura 1). La amplitud del cauce es variable pero no alcanza más de los $2 \mathrm{~m}$, el sustrato es arenoso con guijarros en el origen y posteriormente de cantos rodados y grandes rocas desprendidas de una mina de ónix cercana.

$\mathrm{El}$ agua del arroyo presenta características relativamente constantes a lo largo de todo el año, condiciones alcalinas con una proporción alta de electrólitos y al mismo tiempo muy poco oxígeno disuelto (tabla 1).

\section{Material y método}

Los parámetros químicos como nitrógeno de nitritos, nitratos, amoniacal; ortofosfatos, bióxido de sílice fueron determinados por métodos colorimétricos (Fernández, 1990).

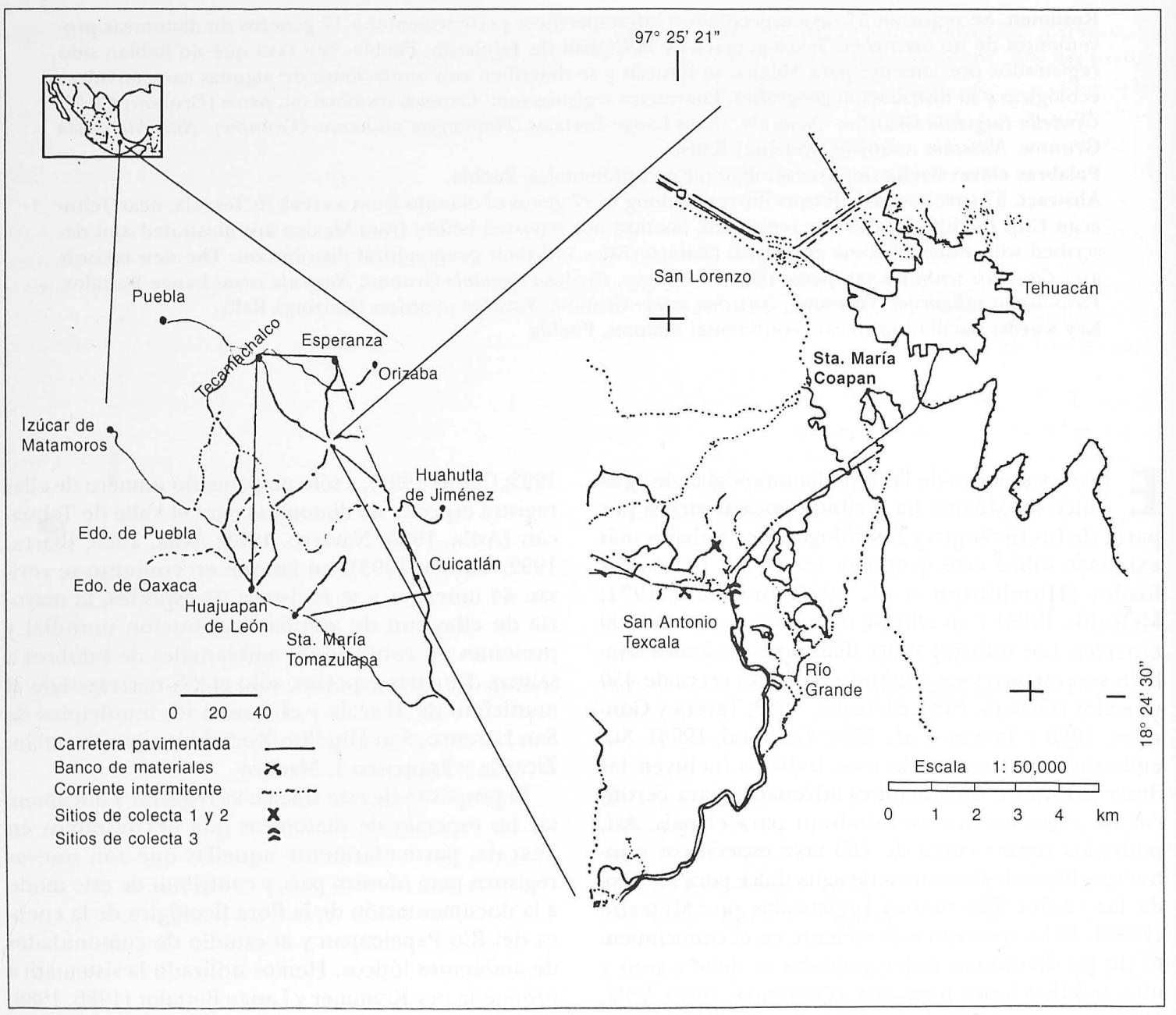

Figura 1. Ubicación geográfica de la zona de estudio. 
Tabla 1. Intervalos de algunos factores ambientales del arroyo de San Antonio Texcala, $n=6$.

$\begin{array}{ll}\mathrm{pH} & 7.0-8.3 \\ \text { Temperatura } & 21.0-26.6{ }^{\circ} \mathrm{C} \\ \text { Oxígeno disuelto } & 0.02-0.05 \mathrm{mg} \mathrm{I}-1 \\ \text { Conductividad } & 2220-4640 \mu \mathrm{S} \mathrm{cm}-1 \\ \text { Dureza total } & 286 \mathrm{meq} \mathrm{I-1} \\ \text { Nitrógeno de Nitratos } & 40-70 \mu \mathrm{g} \mathrm{I}-1 \\ \text { Nitrógeno de Nitritos } & 0-3 \mu \mathrm{g} \mathrm{I}-1 \\ \text { Nitrógeno amoniacal } & 12-43.5 \mu \mathrm{g} \mathrm{l}-1 \\ \text { Ortofosfatos } & 0.0-0.03 \mu \mathrm{gl}-1 \\ \text { Sílice (SiO2) } & 3-13 \mathrm{mg} \mathrm{l-1}\end{array}$

El oxígeno, la temperatura, la conductividad y el $\mathrm{pH}$ fueron registrados in situ con un oxímetro YSI 51b y un potenciómetro-conductímetro Conductronic 2000 respectivamente.

De las muestras colectadas a lo largo del cauce, se analizaron las marcadas con los números 202 - 209 de la colección del Proyecto Flora Ficológica de la cuenca del río Papaloapan del Herbario de la Facultad de Ciencias de la UNAM (FCME - PAP). Estas muestras fueron seleccionadas por constituir crecimientos visibles que representan diferencias en la vegetación algal en el cauce:

FCME - PAP 202. Filamentos epipélicos de Enteromorpha intestinalis $\mathrm{L}$. y Cladophora rivularis (L.) van den Hoek, de color verde oscuro y textura rasposa. Diatomeas epífitas.

FCME - PAP 203. Perifiton de un crecimiento similar y vecino de la muestra PAP 202.

FCME - PAP 204. Crecimientos de Chara globularis Thuillier, fijos al sustrato. Diatomeas perifíticas

FCME- PAP 205. Película mucilaginosa epilítica, guijarros ubicados bajo una caída de agua.

FCME - PAP 206. Filamentos epilíticos de Enteromorpha intestinalis y Cladophora rivularis, de color verde limón y textura rasposa. Diatomeas epífitas

FCME - PAP 207. Crecimientos epilíticos, laxamente adheridos de Cladophora rivularis, Enteromorpha intestinalis y Mougeotia sp. de color verde seco. Diatomeas epífitas.

FCME - PAP 208. Película epilítica de textura suave y lisa de color verde pardusca, compuesta por diatomeas.

FCME - PAP 209. Crecimientos de Chara globularis fijos al sustrato. Diatomeas perifíticas.

La limpieza de la diatomeas se hizo empleando digestión ácida (hirviendo en ácido nítrico por varios minutos y dicromato de potasio) y montadas en $\mathrm{Na}$ phrax.

\section{Resultados}

En el arroyo de Texcala se determinaron 57 taxa pertenecientes a 17 géneros de los órdenes Centrales y Pennales de la Clase Bacillariophyceae. Varias especies han sido encontradas previamente en México pero bajo nombres distintos según el sistema de Krammer y Lange Bertalot (1986 - 1991b). Los taxa encontrados se enlistan en la tabla 2 y para las especies que no han sido registradas para México, se hace una breve descripción de la morfología encontrada en Texcala, su distribución geográfica, algunas características ecológicas y las referencias bibliográficas utilizadas para su determinación.

\section{Achnanthaceae}

Cocconeis scutellum Ehrenberg var. parva (Grunow) Cleve 1895.

= Cocconeis scutellum f. parva Grunow in van Heurck.

Valvas elípticas, de 15.8 a $15.9 \mu \mathrm{m}$ de largo y $10.6 \mu \mathrm{m}$ de ancho. La valva con rafe con área axial lineal, área central pequeña y redondeada; las estrías formadas por areolas redondeadas que presentan una densidad de 8 areolas en las estrías centrales. Estrías paralelas en el centro y radiadas en los ápices, la densidad de las estrías es de 14 en $10 \mu \mathrm{m}$. En la valva sin rafe el área axial es lineal y no se observa área central, estrías paralelas en el centro y curvas en los extremos, con una densidad de 12 en $10 \mu \mathrm{m}$. en una disposición semejante a las de la valva con rafe. Crece como perifiton de algas filamentosas (figura 2a).

Se distingue de la forma nominal por la conspicuidad de las areolas ordenadamente dispuestas de la valva $\sin$ rafe.

Muestras: FCME - PAP 203.

Distribución ecológica: aguas alcalinas, con muy alta conductividad, en zonas costeras, reófilas. En Texcala en aguas alcalinas, muy conductivas. Forma de vida: perifiton. Distribución geográfica: Europa central y Estados Unidos.

Referencias: van Heurck, 1899; Hustedt, 1927 - 1966; Hustedt, 1930; Patrick y Reimer, 1966; Krammer y Lange Bertalot, 1991b.

\section{Naviculaceae}

Navicula recens (Lange-Bertalot) Lange-Bertalot 1985. = Navicula cari var. recens Lange-Bertalot.

Frústulas lanceoladas con los extremos ligeramente rostrados y redondeados de 23.8 a $34.1 \mu \mathrm{m}$ de largo 
y de 5.9 a $11 \mu \mathrm{m}$ de ancho. Area axial lineal, área central marcadamente elíptica. Estrías paralelas en los extremos y radiales en la parte media, con una densidad de 9 en $10 \mu \mathrm{m}$. Se observa un nódulo central grueso, las tres estrías centrales de cada lado son más cortas que las demás, limitadas por estrías fuertemente radiales. Rafe lineal con los extremos distales curvos, en forma de gancho en cada extremo.

Epífitas y perifíticas de algas filamentosas y epilíticas. (figura $2 b$ ).

Se distingue por la densidad y disposición de las estrías centrales, así como por la forma del área central.

Muestras revisadas: FCME - PAP 202, 203, 204, 206, 207, 209.

Distribución ecológica: En grandes ríos, aguas salobres y altamente conductivas. Tolerante a la contaminación orgánica. En Texcala en aguas alcalinas, muy conductivas. Forma de vida: perifiton, epiliton. Distribución geográfica: Asia menor (Jordania), Estados Unidos, Europa central, Jamaica.

Referencias: Krammer y Lange Bertalot 1986.
Cymbella turgidula Grunow in A. Schmidt et al., 1875.

Valvas dorsalmente convexas y ventralmente rectas con los ápices redondeados, ligeramente distinguibles del cuerpo de la valva. Largo de 25.5 a 30.4 $\mu \mathrm{m}$ y ancho de 8.6 a $9.3 \mu \mathrm{m}$; área axial lineal y arqueada, área central orbicular, con dos estigmas ventrales, estrías punteadas radiales a lo largo de toda la valva con una densidad de 11 en $10 \mu \mathrm{m}$. El rafe, en posición ventral, reverso-lateral con los extremos distales curvados dorsalmente y los extremos proximales cercanos y rectos. Perifiton de algas filamentosas (figura 2c).

Se distingue por su talla, la densidad de las estrías, la forma de los ápices y del área central, así como por la presencia de estigmas en la misma.

Muestras: FCME- PAP 203, 204.

Distribución ecológica: arroyos, ríos; bien oxigenadas, indiferente al pH. En Texcala en aguas alcalinas, muy conductivas. Forma de vida: forma crecimientos masivos (matas algales). En Texcala siempre está asociado, sin crecimientos abundantes. Distribución geo-



Figura 2. Nuevos registros de diatomeas para México, a] Cocconeis scutellum var. parva (Grunow) Cleve; b] Navicula recens (Lange Bertalot) Lange Bertalot; c] Cymbella turgidula Grunow; d] Pleurosigma salinarum Grunow; e] Nitzschia calida Grunow; $f$ ] Nitzschia constricta (Kützing) Ralfs. La escala equivale a $10 \mu \mathrm{m}$. 
gráfica: En regiones tropicales (Puerto Rico), centro y este de Estados Unidos, Europa central, India.

Referencias: Hustedt, 1930; Patrick y Reimer, 1975; Sarode y Kamat, 1984; Krammer y Lange Bertalot, 1986.

Pleurosigma salinarum Grunow in Cleve et Grunow 1880.

\section{= Pleurosigma pusillum Grunow.}

Frústulas sigmoides con los ápices agudos, 75.2 a $82.4 \mu \mathrm{m}$ de largo y ancho de 14.5 a $15.7 \mu \mathrm{m}$; área axial sigmoide, área central elíptica, estrías transversales con una densidad de 27 en $10 \mu \mathrm{m}$ y diagonales con una densidad de 20 en $10 \mu \mathrm{m}$ en una inclinación de $60^{\circ}$; el rafe no está colocado simétricamente en la valva, sino desplazado en sus extremos distales hacia el margen valvar. Algas epilíticas (figura 2d).

Se distingue por las características de los ápices y la estriación fina.

Muestra: FCME - PAP 205.

Distribución ecológica: cosmopolita en aguas salobres, salinas y moderadamente saladas. Marismas y arroyos. En Texcala en aguas alcalinas, muy conductivas. Forma de vida: Según la bibliografía es perifítica en Texcala sólo en crecimientos epilíticos. Distribución geográfica: Sureste de Estados Unidos, Europa, India.

Referencias: Hustedt, 1930; Patrick y Reimer, 1966; Cook y Whipple, 1982; Sarode y Kamat, 1984; Krammer y Lange Bertalot, 1986.

\section{Bacillariaceae}

Nitzschia calida Grunow in Cleve et Grunow 1880 = Nitzschia tryblionella var. calida (Grunow) Van Heurck.

Frústula lineal lanceolada, con un margen cóncavo, los extremos son agudos y ligeramente alargados, de 27.7 a $30 \mu \mathrm{m}$ de largo y de 8.9 a $9.2 \mu \mathrm{m}$ de ancho; con 10 fíbulas cortas en $10 \mu \mathrm{m}$, regularmente distribuidas, las estrías son paralelas en el centro y curvas en los ápices, de 32 a 36 en $10 \mu \mathrm{m}$; el rafe es muy evidente, excéntrico, con un nódulo central pequeño poco evidente. En crecimientos epilíticos (figura 2e).

Se distingue por el ancho de las valvas, así como por la distribución y curvatura de las estrías.

Muestra: FCME - PAP 208.

Distribución ecológica: cosmopolitas, muy frecuentes en regiones con agua medianamente o muy con. ductiva, a menudo con otras diatomeas halófilas; en estanques y arroyos eutróficos. También en aguas ligeramente saladas. Forma de vida: en la bibliografía aparece como perifiton, en Texcala en crecimientos epilíticos. Distribución geográfica: Europa, Jamaica, Sudáfrica, Sri Lanka, India.
Referencias: van Heurck, 1899; Hustedt, 1930; Sarode y Kamat, 1984; Krammer y Lange Bertalot, 1988.

Nitzschia constricta (Kützing) Ralfs in Pritchard 1861 non (Gregory) Grunow 1880.

= Synedra constricta Kützing; Tryblionella apiculata Gregory; Nitzschia apiculata (Gregory) Grunow

Frústulas lineales con ambos márgenes cóncavos y los ápices acuminados a subrostrados, de 20 a 37.5 $\mu \mathrm{m}$ de largo y de 4 a $6.2 \mu \mathrm{m}$ de ancho; las fíbulas no son muy evidentes y tienen una densidad de 9 en 10 $\mu \mathrm{m}$, con un nódulo central pequeño; la densidad de las estrías es de 11 a 20 en $10 \mu \mathrm{m}$; un área axial hialina angosta de un ápice a otro. Epifiton y perifiton de algas filamentosas, también en crecimientos epilíticos (figura 2f).

Krammer y Lange Bertalot (1988) registran una densidad de fíbulas mayor.

Se distingue por la forma de los ápices, los márgenes cóncavos, la densidad de estrías y fíbulas y la talla.

Muestras: FCME - PAP 202, 205, 207, 208, 209.

Distribución ecológica: sedimentos de aguas salobres costeras, aguas ricas en electrolitos, $\alpha$ mesosaprobias, en marismas, suelos húmedos de arroyos salobres. Alcalófila a indiferente al $\mathrm{pH}$, arroyos o indiferente a la corriente. Forma de vida: En la bibliografía se registra como perifítica y edáfica. En Texcala es perifítica epífita, y epilítica. Distribución geográfica: Europa central, India, sureste de Estados Unidos, Francia, Irak.

Referencias: como N. apiculata, van Heurck, 1899, Hustedt, 1930; Sieminska, 1964; Hirano, 1973; Lowe, 1974; Starmach, 1980; Germain, 1981; Cook y Whipple, 1982; Sarode y Kamat, 1984. Como N. constricta, Krammer y Lange Bertalot, 1988.

\section{Discusión}

La riqueza de especies de diatomeas del Valle de Tehuacán y en particular del municipio de Texcala es muy alta, pues de la revisión de sólo 8 muestras de esta localidad se registran 57 taxa específicos e infraespecíficos, con 6 que no habían sido reportados antes para México; estos datos son importantes si consideramos que de la revisión de 44 muestras reunidas en 4 trabajos de tesis de licenciatura y maestría, pertenecientes a 6 municipios de este mismo Valle (San Lorenzo, San Hipólito, Xochiltenango, Teotitlán, Zicastla y Francisco I. Madero) se obtuvieron un total de 92 especies; es decir, en Texcala se encuentra representado aproximadamente el $60 \%$ del total de las especies reportadas para todo el Valle de Tehuacán desde 1985. Además, se reafirma la convicción de que la revisión ficoflorística de cualquier localidad no puede darse por agotada, prueba de ello son 
Tabla 2. Diatomeas de Texcala, Puebla

\begin{tabular}{llllllllll}
\hline Especie & \multicolumn{1}{c}{ Muestras } \\
& 202 & 203 & 204 & 205 & 206 & 207 & 208 & 209 & \\
\hline
\end{tabular}

Achnanthes affinis Grunow

A. exigua var. constricta (Torka) Hustedt

A. lanceolata ssp. dubia (Grunow) Lange Bertalot

A. lanceolata ssp. frecuentissima Lange Bertalot

A. lanceolata ssp. lanceolata (Brébisson) Grunow

A. minutissima Kützing

Amphora pediculus (Kützing) Grunow

A. veneta Kützing

Caloneis bacillum Grunow

Cocconeis placentula var. euglypta (Ehrenberg) Grunow

C. placentula var. lineata (Ehrenberg) van Heurck

C. pediculus Ehrenberg

C. scutellum var. parva (Grunow) Cleve

Cymbella affinis Kützing

C. caespitosa (Kützing) Brun.

C. leavis Nägeli

C. microcephala Grunow

C. muellerii Hustedt

C. tumidula Grunow

C. turgidula Grunow

Cylindrotheca gracilis (Brébisson) Grunow

Diploneis subovalis Cleve

D. pseudovalis Hustedt

Denticula kuetzingii Grunow

Epithemia sorex Kützing

Fragilaria brevistriata Grunow

F. Ulna (Nitzsch) Lange Bertalot

F. ulna var. oxyrhynchus (Kützing) van Heurck

Gomphonema clavatum Ehrenberg

G. Iongiceps Ehrenberg

G. minutum (Agardh) Agardh

G. parvulum (Kützing) Kützing

G. ventricosum Gregory

Navicula subminuscula Manguin

N. cari Ehrenberg

N. cryptotenella Lange Bertalot

N. halophila (Grunow) Cleve

N. lacustris Gregory

N. minuscula Grunow

N. orangiana Patrick

$N$. recens Lange Bertalot

N. rhynchocephala Kützing

Nitzschia amphibia Grunow

N. bacillum Hustedt

N. calida Grunow

N. constricta (Kützing) Grunow

$N$. hantzschiana Rabenhorst

N. linearis (Agardh) W. Smith

N. inconspicua Grunow

N. microcephala Grunow

N. communis Rabenhorst

N. palea (Kützing) W. Smith

N. umbonata (Ehrenberg) Lange Bertalot

Rhopalodia gibberula (Ehrenberg) O. Müller

Surirella ovalis Brébisson

Pleurosira leavis (Ehrenberg) Compère

Pleurosigma salinarum Grunow

\begin{tabular}{|c|c|c|c|c|c|c|c|}
\hline 0 & 0 & + & 0 & + & 0 & 0 & + \\
\hline 0 & 0 & 0 & + & 0 & 0 & 0 & 0 \\
\hline 0 & 0 & 0 & + & 0 & 0 & 0 & 0 \\
\hline 0 & + & + & + & 0 & 0 & 0 & 0 \\
\hline 0 & + & 0 & + & 0 & 0 & 0 & 0 \\
\hline 0 & + & 0 & + & 0 & 0 & 0 & 0 \\
\hline+ & + & 0 & 0 & 0 & 0 & 0 & 0 \\
\hline 0 & 0 & + & 0 & + & 0 & + & + \\
\hline+ & 0 & 0 & 0 & 0 & 0 & 0 & 0 \\
\hline+ & + & + & + & 0 & 0 & + & 0 \\
\hline+ & 0 & 0 & 0 & 0 & 0 & 0 & 0 \\
\hline+ & 0 & 0 & 0 & 0 & 0 & 0 & 0 \\
\hline 0 & + & 0 & 0 & 0 & 0 & 0 & 0 \\
\hline+ & 0 & 0 & 0 & 0 & 0 & 0 & 0 \\
\hline 0 & + & 0 & 0 & 0 & 0 & 0 & 0 \\
\hline 0 & + & 0 & 0 & 0 & 0 & 0 & 0 \\
\hline 0 & + & 0 & 0 & 0 & 0 & 0 & 0 \\
\hline 0 & + & 0 & 0 & 0 & 0 & 0 & 0 \\
\hline 0 & + & 0 & 0 & 0 & 0 & 0 & 0 \\
\hline 0 & + & + & 0 & 0 & 0 & 0 & 0 \\
\hline 0 & 0 & 0 & + & 0 & 0 & 0 & 0 \\
\hline 0 & 0 & 0 & + & 0 & 0 & 0 & 0 \\
\hline+ & 0 & 0 & 0 & 0 & 0 & 0 & 0 \\
\hline+ & + & 0 & + & 0 & 0 & 0 & + \\
\hline 0 & 0 & 0 & + & 0 & 0 & 0 & 0 \\
\hline 0 & 0 & 0 & + & 0 & 0 & 0 & 0 \\
\hline+ & + & + & 0 & 0 & 0 & 0 & + \\
\hline 0 & + & 0 & 0 & 0 & 0 & 0 & 0 \\
\hline+ & + & 0 & 0 & 0 & 0 & 0 & 0 \\
\hline 0 & + & 0 & 0 & 0 & 0 & 0 & 0 \\
\hline 0 & 0 & 0 & 0 & 0 & 0 & + & 0 \\
\hline+ & 0 & + & + & + & + & 0 & + \\
\hline+ & 0 & 0 & 0 & 0 & 0 & 0 & 0 \\
\hline+ & + & 0 & + & 0 & 0 & 0 & 0 \\
\hline 0 & + & 0 & + & 0 & 0 & 0 & 0 \\
\hline 0 & + & 0 & 0 & 0 & 0 & 0 & 0 \\
\hline 0 & + & 0 & 0 & 0 & 0 & 0 & 0 \\
\hline+ & 0 & 0 & 0 & 0 & 0 & 0 & 0 \\
\hline 0 & 0 & 0 & + & 0 & 0 & 0 & 0 \\
\hline+ & 0 & 0 & 0 & 0 & 0 & 0 & 0 \\
\hline+ & + & + & 0 & + & + & 0 & + \\
\hline 0 & + & 0 & 0 & 0 & 0 & 0 & 0 \\
\hline+ & + & + & + & 0 & 0 & 0 & 0 \\
\hline 0 & 0 & 0 & + & 0 & 0 & 0 & 0 \\
\hline 0 & 0 & 0 & 0 & 0 & 0 & + & 0 \\
\hline+ & 0 & 0 & + & 0 & + & + & + \\
\hline 0 & + & 0 & 0 & 0 & 0 & 0 & 0 \\
\hline 0 & 0 & 0 & + & 0 & 0 & 0 & 0 \\
\hline 0 & + & 0 & + & 0 & 0 & 0 & 0 \\
\hline 0 & + & + & + & 0 & + & + & 0 \\
\hline 0 & + & 0 & + & 0 & 0 & 0 & 0 \\
\hline 0 & + & + & + & 0 & 0 & 0 & + \\
\hline 0 & + & 0 & 0 & 0 & 0 & 0 & + \\
\hline 0 & 0 & 0 & + & 0 & 0 & 0 & 0 \\
\hline+ & + & 0 & 0 & 0 & 0 & 0 & 0 \\
\hline 0 & 0 & 0 & + & 0 & 0 & 0 & 0 \\
\hline 0 & 0 & 0 & + & 0 & 0 & 0 & 0 \\
\hline
\end{tabular}


los 6 registros nuevos para México y la modificación constante de la flora de la localidad (compárense los datos de Ávila, 1985,1989 y la tabla 2). Las especies encontradas representan formas de vida perifíticas y/ o epifíticas, y responden a ambientes reófilos, altamente conductivos, características que están bien representadas en el arroyo de San Antonio Texcala. Las condiciones físicas consideradas (tabla 1) son constantes a lo largo de toda la localidad, no obstante, ninguno de los 57 registros que se enlistan presentan poblaciones con una frecuencia del $100 \%$, es decir, ninguna habita en toda la localidad de colecta.

De las especies no registradas previamente, Cymbella turgidula amplía la distribución registrada para las condiciones de oxígeno, ahora presente en ambientes muy o poco oxigenados y su forma de vida formadora de matas algales macroscópicas y perifítica, como un organismo aislado. Sin embargo, ésta es una especie con una gran variabilidad morfológica y Hustedt (1939), Patrick y Reimer (1975) y Krammer y Lange Bertalot (1986) tienen dudas si los registros que se han hecho previamente en el mundo corresponden al mismo taxón. Pleurosigma salinarum y $\mathrm{Ni}$ tzschia recens se han registrado en la bibliografía como perifiton y edáficas en marismas, mientras que en Texcala forman parte de crecimientos epilíticos. Sin embargo, todas las especies que forman los crecimientos mencionados también se han registrado previamente como perifíticas o epífitas. De esta manera, la condición epilítica en Texcala quizá puede matizarse al considerar la escala de los crecimientos que pueden alcanzar cerca de $0.5 \mathrm{~m}^{2}$ en forma continua y cubrir áreas pedregosas más amplias de manera discontinua en los márgenes del arroyo. Cabría preguntarse si unas especies son perifiton de las otras y la condición epilítica no es determinante como forma de vida.
Otra característica que llama la atención es que, en general las especies observadas presentan tallas más pequeñas que las anotadas por la bibliografía, o bien se ubican entre los niveles mínimos registrados. Esta observación también fue hecha por Tavera et al., (1994) para las especies de diatomeas que habitan en la Sierra de Mixes y Juárez, Oaxaca; estos autores consideran esta disminución generalizada en la talla de especies que son de distribución templada, debida a una acción combinada de la temperatura del agua y la conductividad. Las especies que se encuentran en Tehuacán tienen un amplio espectro ecológico y las tallas mínimas pueden deberse a algunos efectos de la salinidad en las tasas de reproducción sexual y asexual, que podrían restablecer las tallas máximas de las diatomeas. Sin embargo no existen datos de reproducción y lo anterior deberá corroborarse con cultivos dedicados a ello.

Considerando los registros previos de la flora diatomológica es indispensable que las especies que sean observadas en México estén debidamente documentadas, pues el registro de las especies sólo por su nombre no permitirá evaluar la ficoflora dulceacuícola, ni la ecología de las comunidades algales; sobre todo, es necesario conocer bien a las especies y sus variantes morfológicas en México. Una mala determinación no puede ser evaluada si no tiene los datos mínimos, especialmente en el caso de los microorganismos con los que no se cuenta con material de herbario accesible y cuya variación morfológica no está debidamente documentada para todos los países.

\section{Agradecimientos}

La Dra. Rosa Luz Tavera leyó críticamente el manuscrito, agradecemos sus sugerencias que lo mejoraron sustancialmente.

Tabla 3. Condiciones de hábitat y formas de vida registradas por la literatura para las especies que son nuevos registros para México.

\begin{tabular}{|c|c|c|c|c|}
\hline Especie & $\begin{array}{l}\text { nivel } \\
\text { oxigénico }\end{array}$ & $\begin{array}{l}\text { nivel de } \\
\text { conductividad }\end{array}$ & $\begin{array}{l}\text { tipo de } \\
\text { ambiente }\end{array}$ & $\begin{array}{l}\text { Forma } \\
\text { de vida }\end{array}$ \\
\hline Cocconeis scutellum var. parva & alto & alto & reófilo & perifiton \\
\hline Cymbella turgidula & alto & alto & reófilo & matas, perifiton \\
\hline Navicula recens & alto & alto & reófilo & $\begin{array}{l}\text { perifiton, epifiton, } \\
\text { epiliton }\end{array}$ \\
\hline Pleurosigma salinarum & alto & alto & reófilo & perifiton, epiliton \\
\hline Nitzschia calida & alto & alto & reófilo & perifiton, epiliton \\
\hline Nitzschia constricta & alto & alto & reófilo & $\begin{array}{l}\text { epifiton, perifiton, } \\
\text { epiliton }\end{array}$ \\
\hline
\end{tabular}




\section{Literatura citada}

Ávila-Nava,. J. 1985. Ficoflora manifiesta del suelo del Valle de Tehuacán, Puebla. México. Tesis de Licenciatura Facultad de Ciencias. UNAM. México. 147 p.

Ávila-Nava, J. 1989. Ficoflora potencial de suelo húmedo del Valle de Tehuacán, Puebla. Tesis de Maestría Facultad Ciencias. UNAM. México. 93 p.

Bradbury, J. P. 1971. Paleolimnology of lake Texcoco, México. Evidence from Diatoms. Limnology and Oceanography. 16:180-200.

Cantoral- Uriza, E.A. 1990. Ficoflora de ambientes lénticos en la Localidad El Salto, en la región de la Huasteca Potosina. Tesis de Licenciatura Facultad de Ciencias. UNAM. México. 139 p.

Cantoral-Uriza, E.A. 1993. Ecología de comunidades algales del sistema hidrológico El Salto, en La Huasteca Potosina. Tesis de Maestría Facultad de Ciencias, UNAM México. 97 p.

Cantoral-Uriza y G. Montejano-Zurita, 1993. Las algas de la localidad 'El Salto' (San Luis Potosí, México): un ejemplo de estudios florísticos en ambientes cambiantes. Boletín de la Sociedad Botánica de México 53:3-20.

Carmona-Jiménez, J. 1990. Ficoflora de manantiales de la Huasteca Potosina, México. Tesis de Licenciatura Facultad de Ciencias. UNAM. México. 105 p.

Cook, L. L. and S. A. Whipple. 1982. The distribution of edaphic diatoms along environmental gradients of a Louisiana salt marsh. Journal of Phycology 18(1):64-71.

Cuesta- Zarco, I. J. 1993. Ecología de la ficoflora de los Manantiales de San Lorenzo, Tehuacán, Puebla. Tesis de Licenciatura Facultad de Ciencias. UNAM. México. 119 p.

Fernández-Villagómez, G. 1990. Manual de laboratorio de Química de agua. Facultad de Ingeniería. UNAM.

Germain, H. 1981. Flore des Diatomées. Diatomophycees. Eux douces et saumâtres du Massif Armoricain et des contrée voisines d Europe Occidentale. Paris. Société Nouvelle des Éditions Boubée. Francia.

Hirano, M. 1973. Freshwater algae from Mesopotamia. Contributions of the Biology Laboratory. Kyoto University. 24(2):105-19.

Hustedt, F. 1927-1966. Die Kiesenalgen Deutschland, Österreich und der Schweiz mit Berücksichtigungg der übringen Lander Europas sowie der angrenzenden Meeresgebiete. En: L. Rabenhort s Kryptogmen-Flora von Deutschland, Osterreich und der Schweiz. 7:Teil 1-3, Leipzig. Reprint 1971 by Johnson Reprint Corporation, New York.USA.

Hustedt, F. 1930. Bacillariophyta (Diatomeae). En: A. Pascher (Ed.), Die Süsswasser-flora Mitteleuropas, Heft. 10, Jena. Verlag von Gustav Fischer.

Hutchinson, G.E.; R. Patrick. and E.S. Deevey. 1956. Sediments of Lake Pátzcuaro, Michoacán, México. Bulletin of the Geological Society of America 67:1491-1504, 2 figuras.
Ibarra-Vázquez, C. 1992. Flora Diatomológica de Texcala, Tehuacán, Puebla. Tesis de Licenciatura Facultad de Ciencias. UNAM. México. 80 p.

Krammer, K. und H. Lange-Bertalot. 1986. Bacillariophyceae. Teil 1: Naviculaceae. Band 2/1. Die Susswasserflora von Mitteleuropa, Begr. Pascher, A.; Ettl, H.; Gerloff, J.; Heynig, H.; Mollenhauer, D. (Hrsg.), Sttutgart. Gustav Fischer Verlag.

Krammer, K. und H. Lange-Bertalot. 1988. Bacillariophyceae. Teil 2: Bacillariophyceae, Epithemiaceae, Surirellaceae. Band 2/2. Die Susswasserflora von Mitteleuropa, Begr. Pascher, A.; Ettl, H.; Gerloff, J.; Heynig, H.; Mollenhauer, D. (Hrsg.), Sttutgart. Gustav Fischer Verlag.

Krammer, K. und H. Lange-Bertalot. 1991a. Bacillariophyceae. Teil 3. Centrales, Fragillariaceae. Eunothiaceae. Band 2/3. Die Susswasserflora von Mitteleuropa. Begr. Pascher, A.; Ettl, H.; Gerloff, J.; Heynig, H.; Mollenhauer, D. (Hrsg.), Sttutgart. Gustav Fischer Verlag.

Krammer, K. und H. Lange-Bertalot. 1991b. Bacillariophyceae Teil. 4, Achnanthaceae, Kritische Ergõnzungen zu Navicula (Lineolatae) und Gomphonema. Gesamtliteraturverzeichnis Teil 1-4. Die Susswasserflora von Mitteleuropa. Begr. Pascher, A.; Ettl, H., Gerloff, J., Heynig, H., Mollenhauer, D. Sttutgart. Gustav Fischer Verlag.

Lowe, R. L. 1974. Environmental requirements and pollution tolerance of freshwater diatoms. Environmental Monitoring Series. Cincinnati, Ohio, USA. National Environmental Research Center, EPA-670/4-74-005. U.S. Environmental Proteccion Agency.

Metcalfe, S.E. 1985. Late Quaternary environments of central México: A diatom record. Ph.D. Diss. Hertford College and the School of Geography, Oxford, USA. $565 \mathrm{p}$.

Metcalfe, S.E. 1988. Modern diatom assembleges in central Mexico: the role of water chemistry and other environmental factors as indicated by TWISPAN and DECORANA. Freshwater Biology 19:217-33.

Navarro-Jiménez, L.E. 1988. Un estudio tónico de Rhoicosphenia curvata (Kütz.) Grunow ex Rabenh. var. curvata en el Valle de Tehuacán, Puebla. Tesis de Licenciatura Facultad de Ciencias, UNAM. México. 120 p.

Ortega, M.M. 1984. Catálogo de algas continentales recientes de México.unam. México.

Patrick, R. and Ch. W. Reimer. 1966. The Diatoms of the United States. Part I. Monographs of the Academy of Natural Sciences of Philadelphia. USA.

Patrick, R. and Ch. W. Reimer. 1975. The Diatoms of the United States. Part II. Monographs of the Academy of Natural Sciences of Philadelphia. USA.

Quintana-González, G.1961. Estudio de las diatomeas fósiles de México en el microscopio electrónico y en el espectrofotómetro de rayos X. Tesis de Licenciatura. Facultad de Ciencias, UnAm. México. 79 p.

Rzedowski, J. 1978. Vegetación de México. LIMUSA. México. Sarode, P. T. and N. D.Kamat. 1984. Freshwater diatoms of 
Maharashtra. Maharashtra, India. Saikripa Prakashan.

Sheath, R. G. and K. M. Cole. 1992. Biogeography of stream macroalgae in North America. Journal of Phycology $28(4): 448-60$.

Sieminska, J. 1964. Bacillariophyceae Okrzemki. En: Starmach, K. (Ed.) Flora Slodkowodna Polski. Tom 6, Warzawa. Polska Akademia Nauk.

Starmach, K. 1980.Communities of algae in Frog Spawn. Acta Hydrobiol. 22(2): 127-145

Tavera-Sierra, R.L. y González-González, J. 1990. Caracterización ficoflorística de los paredones de la Sierra de Juárez, Oaxaca. Importancia de las formas de crecimiento algales en la tipificación de un ambiente. Boletín de la Sociedad Botánica de México 50: 121-133.

Tavera-Sierra, R.L., Elster, J. and Marvan, P. 1994. Diatoms from Papaloapan basin communities, Mexico. Archiv für Hydrobiologie/ Supplemnt 104, Algological Studies 74:3565.

Valadez-Cruz, F. 1992. Flora ficológica de ambientes lóticos de la cuenca baja del Río Amacuzac, Morelos. Tesis de Licenciatura Facultad de Ciencias, UNAM. México. 239 p. Van Heurck, H. 1899. Traité des Diatomeés.Bruxeles. J.R. Hausen. 